\title{
Evaluation of Upper Limb Muscle Load in Lower-Limb Amputees during Ladder Climbing
}

\author{
Kisuke Iwasaki, Gen Okuya, Yoshiki Nishisako, \\ Naoki Chijiwa, Hisashi Arizono, Chikamune Wada
}

\begin{abstract}
In recent years, the popularity of sport climbing competitions has increased considerably. Currently, not only non-disabled people but also people with disabilities are interested in sport climbing. However, the evaluation and guidance in climbing competitions are often subjective, and there are individual differences in the progress of sport climbing performance.

Additionally, the current training methods have been developed for non-disabled people, and it is not known whether they are suitable for people with disabilities. If an objective evaluation method is established, it will be possible to propose rational and effective teaching methods for sport climbing participants. Therefore, the goal of this investigation was to develop an objective training method for climbing aimed at lower-limb amputees.

We studied subjects' climbing a ladder that imitated the wall-climbing process and compared it with normal conditions and dead-point conditions. At that time, as a result of measurement of the upper limb muscle, it was suggested that both the nonamputeeand the lower-limb amputee can reduce the load on the upper limb musclesduring thedead-point condition.
\end{abstract}

Index Terms - climbing, human engineering, adapted sports, motion analysis, musculoskeletal simulator

\section{INTRODUCTION}

Sport climbing, which became one of the official sports for the 2020 Tokyo Olympics, has recently increased its number of participants across the globe. The population of climbers is reported to be approximately 500,000 in Japan, 25,000,000 people worldwide [1-2].In addition, as the number of climbing gyms and the popularity of climbing competitions continue to increase, itis expected that the scale of future

Kisuke Iwasaki, Graduate School of Life Science and SystemsEngineering, Kyushu Institute of Technology, Hibikino 2-4 Wakamatsu-ku, Kitakyushu Fukuoka 808-0196, Japan.

Gen Okuya, ARIZONO ORTHOPEDIC SUPPLIES CO., LTD., Higashida 1-7-5 Yahatahigashi-ku, Kitakyushu Fukuoka 805-8538, Japan.

Yoshiki Nishisako, Graduate School of Life Science and Systems Engineering, Kyushu Institute of Technology, Hibikino 2-4 Wakamatsu-ku, Kitakyushu Fukuoka 808-0196, Japan.

Naoki Chijiwa, ARIZONO ORTHOPEDIC SUPPLIES CO., LTD., Higashida 1-7-5 Yahatahigashi-ku, Kitakyushu Fukuoka 805-8538, Japan.

Hisashi Arizono, ARIZONO ORTHOPEDIC SUPPLIES CO., LTD., Higashida 1-7-5 Yahatahigashi-ku, Kitakyushu Fukuoka 805-8538, Japan.

Chikamune Wada, Graduate School of Life Science and SystemsEngineering, Kyushu Institute of Technology, Hibikino 2-4 Wakamatsu-ku, Kitakyushu Fukuoka 808-0196, Japan. competitions will expand [1]. Furthermore, it is assumed that many people consider climbing as an adapted sport. There are three categories in a para-climbing competition, visual impairment / disconnection / neuropathy, and classification according to the degree of disability is carried out among them [3]. These also mean that there are environments in which various individuals have the ability to compete, making it easy for those with disabilities to start sport climbing.Therefore, not only non-disabledpeople but also those with disabilities are interested in sport climbing.

However grade setting, which indicates the degree of difficulty of climbing, is done subjectively in the evaluation of climbing competition [4], and guidance is often given by subjective experts. Therefore, objective teaching methods for various competitors have not been established, and there are problems in the improvement of performance across different climbing grades. There is a possibility that a rational and effective teaching method can be created if an objective evaluation method is established. Furthermore, it is conceivable that the present subjective teaching method does not conform to the guidance to people with disabilities such as lower-limb amputees. In the case of teaching climbing to the lower-limb amputee, although it is said that it is difficult to clear with the same guidance as a nonamputeeto climb the task of high difficulty, the objective and rational teaching method no proposal has been made [5].

Generally, in climbing, it has been demonstrated that the exercise load on the upper limb muscles is high [6, 7]. Unlike nonamputees, lower-leg amputees cannot use both feet during climbing; therefore, it is expected that the exercise load on the upper limb will increase, and we have recently reported that the exercise load of upper limb became bigger and tired earlier for the lower-limb amputee than that for the nonamputee [8].Incidentally, in climbing, competition is carried out by using not only a just basic motion that climbs up to the next hold (from now on called Normal) but also a technicalmotion called "moves." These moves are used to achieve complicated tasks during climbing and to reduce the load on competitors [9]. This time, we focus on the dead point (from now on called Dead) which can be used for lower-limb amputees among several move [5].Figure 1 (A) shows an explanation of Normal.Figure 1 (B) shows an explanation of Dead. According to the current instruction methodology, Dead, which is a technical move, is the state where the player is holding the hold with both hands and it is used when the player cannot hold the next Hold by releasing one hand. In addition, by instantly attracting the body to the 


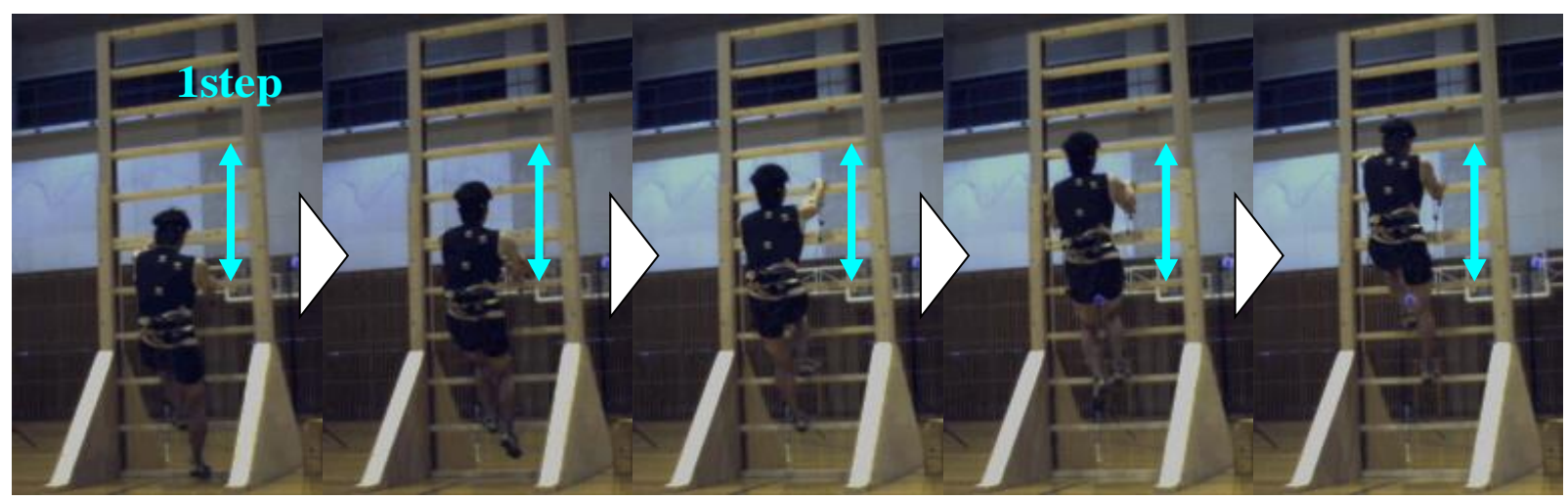

Fig. 1 (A) An explanation of Normal condition

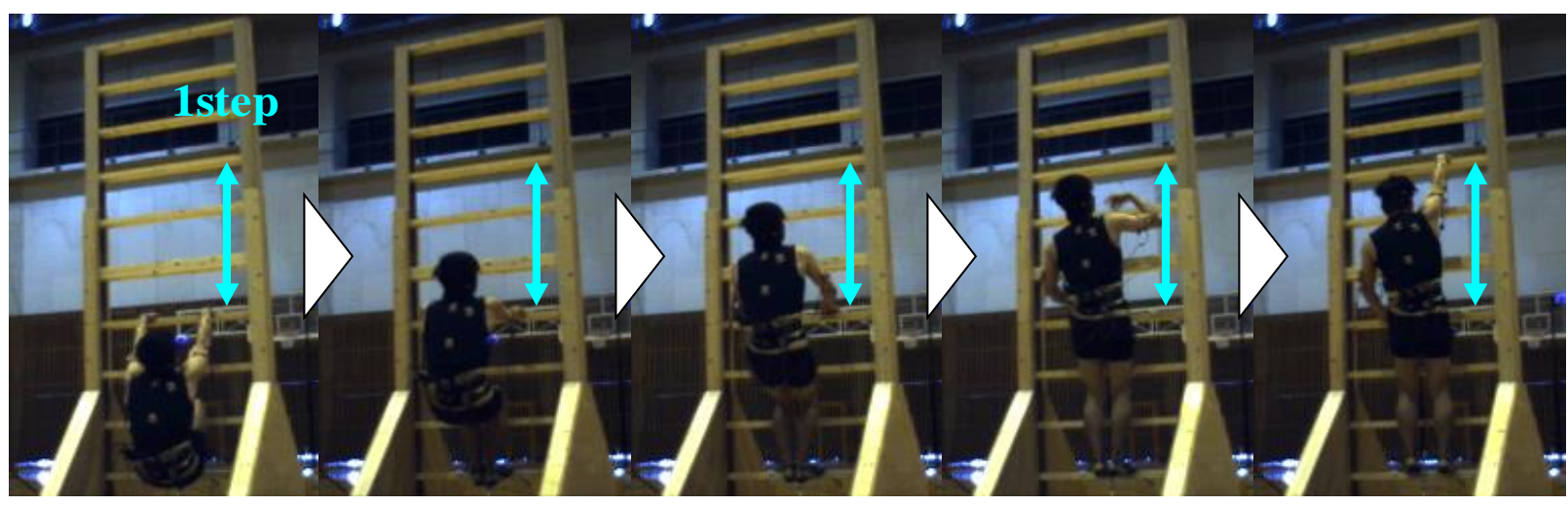

Fig. 1 (B) An explanation of Dead condition

wall with both hands (2nd photo in Fig. 1 (B)), going to take the next hold at the moment when the hand no longer feels weight (3nd photo in Fig. 1 (B)), we create a state of zero speed, that is, zero gravity (4th photo in Fig. 1 (B)) [10, 11, 12]. Based on this fact, if we used Dead during bouldering, we thought that we could reduce the load placed on the muscles by not using the muscle strength needed to climb when we investigated the Dead.

The final goal of this research was motion analysis based on biomechanical knowledge, the establishment ofan objective evaluation method, and the proposal of teaching methodsfor bouldering.In this paper, we aimed to reduce the exercise load of upper limb muscle during climbing for the lower-limb amputee, and by examining and comparing Normal and Dead. There are currently no reports on the effects of Dead on upper limbs,even in nonamputees. Therefore, first, we analyzed the effects of Dead on upper limbs fornonamputee and,then,analyzedthat for a lower-limb amputee.

\section{EXPERIMENT}

In the present investigation, weexperimented with the ladder-climbing motion (height 3,180 mm, width 1,000 mm, number of steps 10). Two subjects participated in the experiment: one nonamputee (subject A, $1.75 \mathrm{~m}, 64 \mathrm{~kg}$,seven years and a half) and onelower-limb amputee (left femoral amputation, subject $\mathrm{B}, 1.71 \mathrm{~m}, 55 \mathrm{~kg}$, oneyear and a half). The subjects were asked to perform two types of climbing,ten times each: climbing one step at a time (Normal condition) and a special climbing method (Dead condition).In this issue, electromyography (EMG) was used to evaluate a load of upper limb muscle during Normal and Dead, three-dimensional motion analysis system to evaluate climbing motion, and the musculoskeletal simulation was used for more detailed analysis of muscle activity and motion. A description of each system is given below.

The surface electromyogram (sEMG) of the upper limbs were measured using wireless EMG Logger II (Logical Product Corporation) to evaluate muscle activity. The left and right sEMG at a total of 4 points of sEMG were measured from the biceps brachii muscle of the left and right arm and triceps brachii muscle of the left and right arm. The biceps brachii muscle is the elbow joint flexion muscle of the upper limb, the triceps brachii muscle is the elbow joint extention muscle of the upper limb. Figure 2 shows the measured muscle position. The sEMG was measured from the start of climbing until when both hands were matched at the top end of the ladder. Next, the sEMG of each part was integrated and normalized totime in motion for each trial.The \%MVC was calculated by performing normalization at Maximum Voluntary Contraction (MVC) for each muscle and normalized by the length of time for each trial.

Furthermore, a three-dimensional motion analysis system (manufactured by OptiTrack) was used to measure the data of the climbing motion to read into the model of the musculoskeletal simulation. According to the Plug-in-Gait marker set, a reflection marker of 41 points (lower-limb amputee is 32 points) was applied to the subject's body surface, and the measurement was performed at a sampling frequency of $100 \mathrm{~Hz}$. Using the AnyBody Modeling System (manufactured by AnyBody Tecnology) [13,14] of the 
Table 1 The sEMG of the upper limb of the subject A Normal condition and Dead condition

\begin{tabular}{|c|c|c|c|c|}
\hline \multirow{2}{*}{ Subject } & \multirow{2}{*}{ Kind of Muscles } & \multicolumn{2}{|c|}{ Normalized sEMG $[\mu \mathrm{V}]$} & \multirow{2}{*}{$\begin{array}{l}\text { Significant } \\
\text { difference }\end{array}$} \\
\hline & & Normal condition & Dead condition & \\
\hline \multirow{4}{*}{ A } & $\begin{array}{l}\text { Right biceps brachii } \\
\text { muscle }\end{array}$ & $174.24 \pm 44.77$ & $130.15 \pm 16.12$ & $* *$ \\
\hline & $\begin{array}{l}\text { Right triceps brachii } \\
\text { muscle }\end{array}$ & $66.77 \pm 13.52$ & $61.43 \pm 18.91$ & NS \\
\hline & $\begin{array}{l}\text { Left biceps brachii } \\
\text { muscle }\end{array}$ & $255.35 \pm 65.30$ & $64.09 \pm 21.15$ & $* *$ \\
\hline & $\begin{array}{l}\text { Left triceps brachii } \\
\text { muscle }\end{array}$ & $55.74 \pm 4.42$ & $48.98 \pm 15.64$ & NS \\
\hline
\end{tabular}

Mean \pm S.D. $\quad *: \mathrm{p}<0.05 \quad * *: \mathrm{p}<0.01$

Table 2 The sEMG of the upper limb of the subject B Normal condition and Dead condition

\begin{tabular}{|c|c|c|c|c|}
\hline \multirow{2}{*}{ Subject } & \multirow{2}{*}{ Kind of Muscles } & \multicolumn{2}{|c|}{ Normalized sEMG $[\mu \mathrm{V}]$} & \multirow{2}{*}{$\begin{array}{l}\text { Significant } \\
\text { difference }\end{array}$} \\
\hline & & Normal condition & Dead condition & \\
\hline \multirow{4}{*}{ B } & $\begin{array}{l}\text { Right biceps brachii } \\
\text { muscle }\end{array}$ & $91.90 \pm 12.90$ & $68.08 \pm 11.98$ & $* *$ \\
\hline & $\begin{array}{l}\text { Right triceps brachii } \\
\text { muscle }\end{array}$ & $29.59 \pm 2.64$ & $40.31 \pm 4.86$ & $* *$ \\
\hline & $\begin{array}{l}\text { Left biceps brachii } \\
\text { muscle }\end{array}$ & $85.93 \pm 15.75$ & $35.08 \pm 9.96$ & $* *$ \\
\hline & $\begin{array}{c}\text { Left triceps brachii } \\
\text { muscle }\end{array}$ & $24.89 \pm 2.55$ & $52.30 \pm 10.06$ & $* *$ \\
\hline
\end{tabular}

Mean \pm S.D. $*: \mathrm{p}<0.05 \quad * *: \mathrm{p}<0.01$

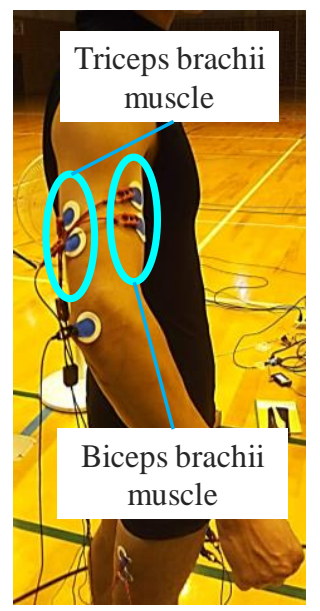

Fig. 2 The measured muscle position
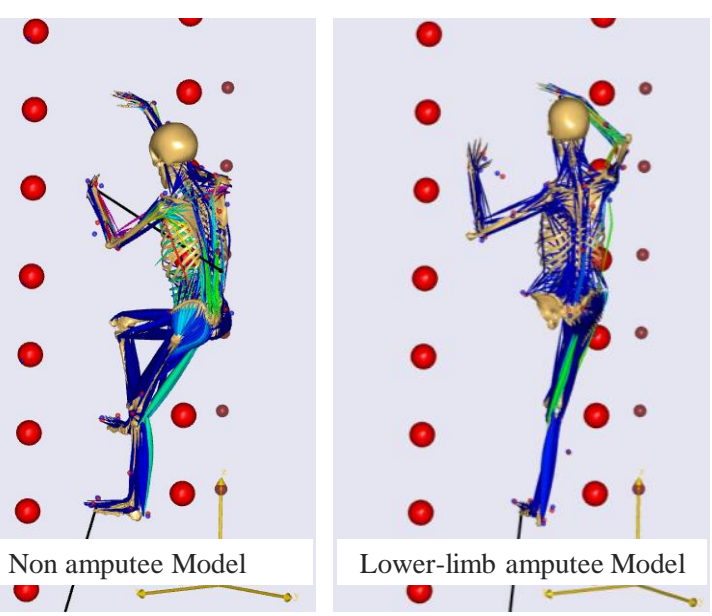

Fig. 3 Nonamputee and Lower-limb amputee Model

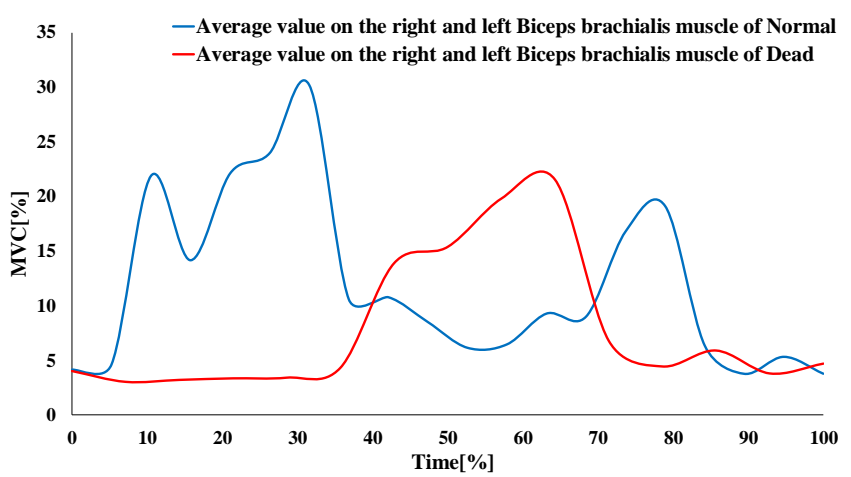

Fig.4 The biceps brachialis muscle in Normal condition and Dead condition of Subject A 


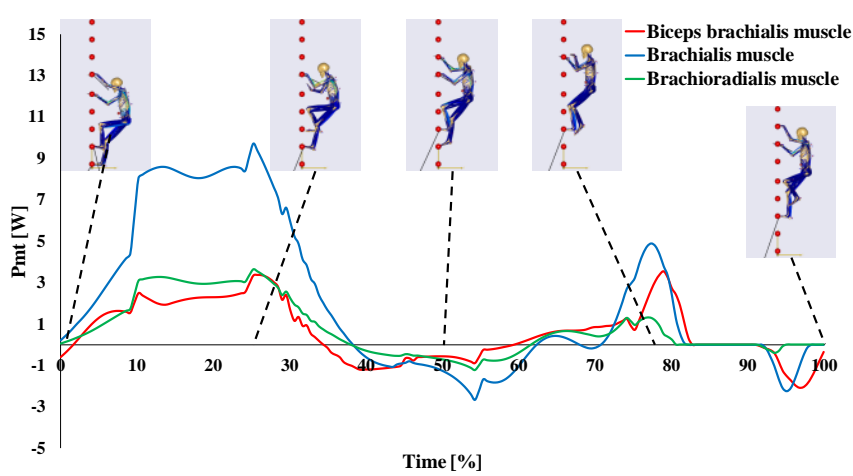

Fig.5Muscle activity of the left elbow joint flexion muscle group in Normal condition of subject A

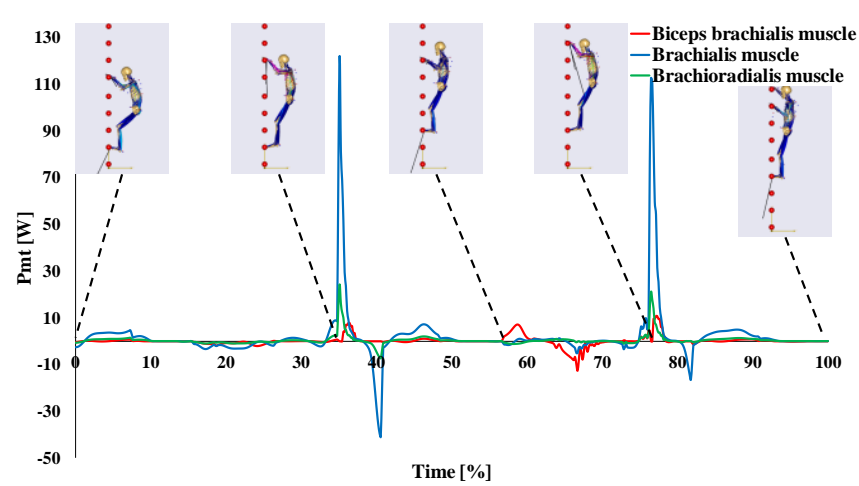

Fig.7 Muscle activity of the right elbow joint flexion muscle group in Normal condition of subject B

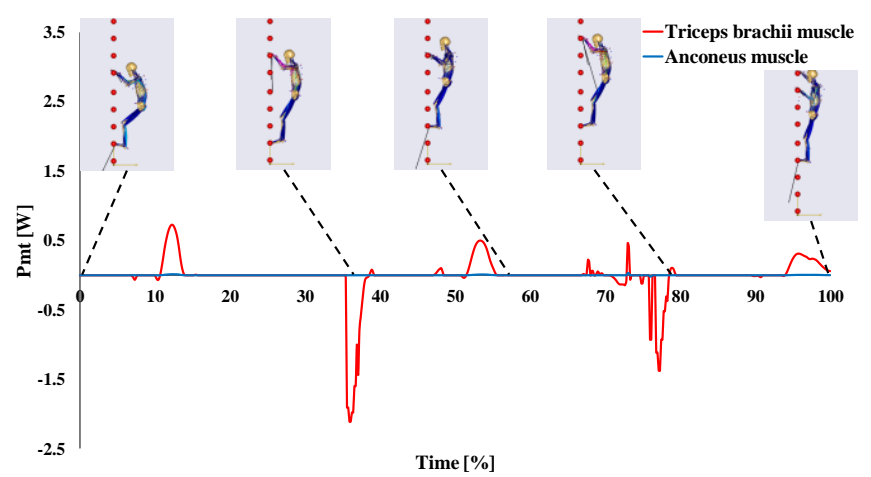

Fig. 9 Muscle activity of the right elbow joint flexion muscle group in Dead condition of subject B

musculoskeletal simulator, the nonamputeewas analyzed by a whole-body model, and the lower-limb amputee

wereanalyzed by a model lacking the left lower limb. Figure 3 shows the respective models.

As an evaluation method, the Wilcoxon signed-rank test was performed between each trial to test for a significant difference. In this study, the step from grounding the foot to the ladder to hanging a hand on the ladders next rung was taken as one step. Then, during the Normal step, it was set as a test section tailored to one step of Dead. In addition, for detailed consideration based on the results, changes in muscle contraction using a musculoskeletal simulator were analyzed. Muscle activity includes agonist and antagonist, fixator (stabilizer), synergist, relaxation, and biarticular muscle. When joint action occurs due to the concentric contraction of one muscle, the muscle is called the agonist, and the antagonist often performs eccentric contraction that adjusts it

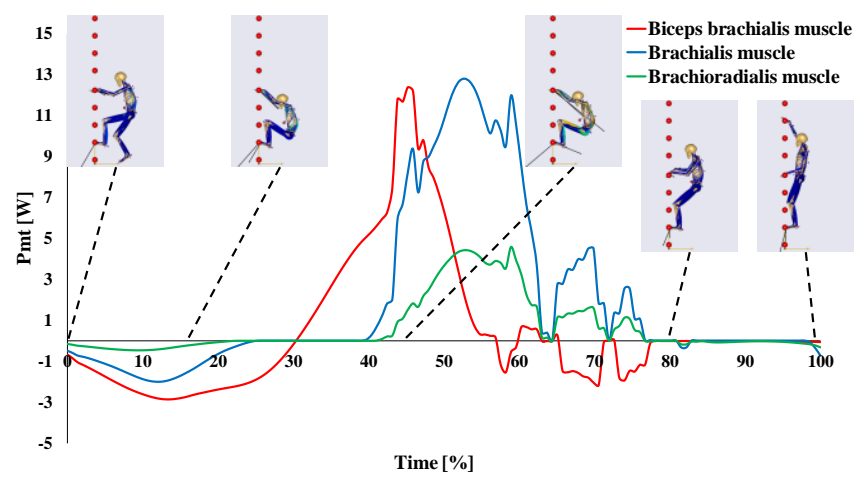

Fig. 6Muscle activity of the left elbow joint flexion muscle group in Dead conditionof subject A

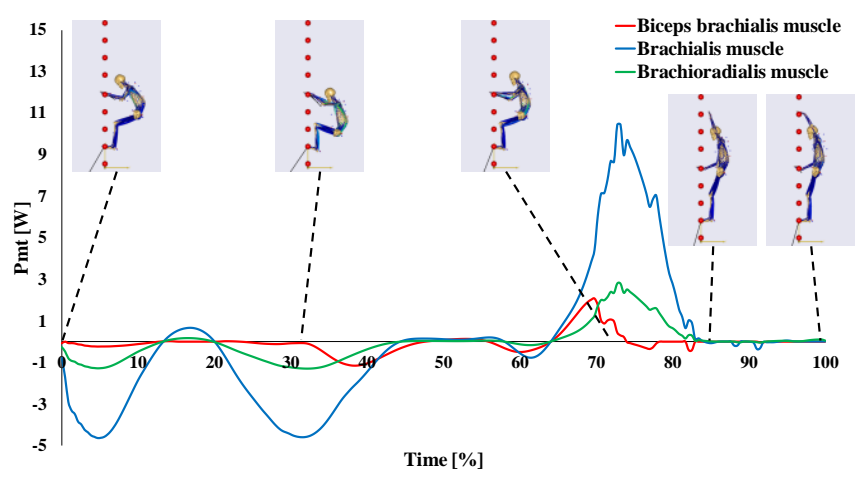

Fig. 8 Muscle activity of the right elbow joint extension muscle groupin Normal condition of subject B

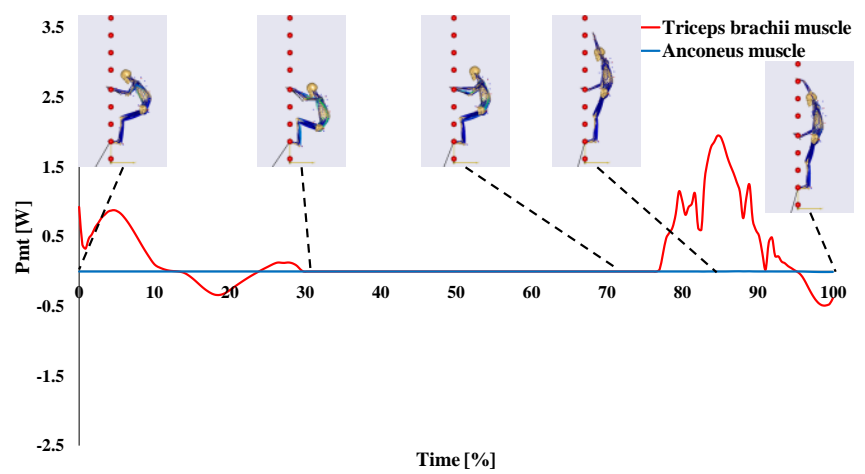

Fig. 10 Muscle activity of the right elbow joint extension muscle group in Normal condition of subject B

according to the change in the speed and intensity of the movement by the agonist [15]. However, in muscle activity, it whether the muscle works as the agonist or as an antagonist cannot be determined. Therefore, we performed a power muscle-tendon, (from now on called Pmt) assessment. The value of Pmt is the power that acts on the skeleton, indicating concentric contraction if it is positive and eccentric contraction if it is negative. During a muscle contraction, an eccentric contraction is known to have a higher muscle load than concentric contraction [16]. Therefore, in this study, by evaluating the temporal change of the muscle contractionwhile climbing the ladder, the difference in muscle load by the trial was evaluated.

\section{RESULTS}

Figure 4 shows the average value of sEMG for one step of the right and left biceps brachialis muscle during a Normal 
condition, and Dead condition of Subject A. The vertical axis represents MVC [\%], and the horizontal axis represents one step period [\%] of climbing. From the results in Fig. 4, comparing the muscle activity of the biceps brachialis muscle in each trial shows that the Dead condition had a shorter duration of muscle activity time than the Normal condition did.

Figure 5-10 shows the results of the muscle activity of subjects A and B. The vertical axis represents Pmt [W], and the horizontal axis represents one step period [\%] of climbing in Fig.5-10. The arm that analyzed the muscle activity was opposite to the arm on which the hand was placed at the time of Dead, that is, the arm on the side that supports the body.

\section{A. Nonamputee (Subject A)}

Table 1 shows the average value and standard deviation in the sEMG of the upper limb of the subject A Normal condition and Dead condition and the results of the test. Based on the results in Table 1, when comparing the sEMG in each trial, the Dead conditionwassignificantly smaller for the biceps brachialis muscle (elbow joint flexion muscle).

Muscle activity of the left elbow joint flexion muscle group (biceps brachialis muscle, brachialis muscle, brachioradialis muscle) in Normal condition of subject $\mathrm{A}$ is shown in Fig. 5, muscle activity of the left elbow joint flexion muscle group in Dead conditionis shown in Fig. 6. Comparing the results of Pmt in Fig. 5 and 6, the muscle activity of the elbow joint flexion muscle group for approximately the same periodis performed in both conditions, and in Dead condition the load was not considered to be small.

\section{B. Lower-limb amputee (Subject B)}

Table 2 shows the average value and standard deviation in the sEMG of the upper limb of subject B in the Normal condition and Dead condition. According to the results in Table 2, when sEMG in each trial is compared, the Dead conditionwassignificantly smaller against the biceps brachialis muscle (elbow joint flexion muscle). However,when compared to the brachial triceps muscle (elbow joint extension muscle) is significantlygreater.

Figure 7 shows muscle activity of the right elbow joint flexion muscle group in Normal condition of subject B. Figure 8shows the muscle activity of the extension muscle group (triceps brachii muscle, anconeus muscle). Figure 9shows the muscle activity of the right elbow joint flexion muscle group of subject B in the Dead condition.Figure 10shows the muscle activity of the extension muscle group. In addition, from the results of Pmt concerning Figs. 9 and 10 the muscle activity for the eccentric contraction of the elbow joint extension muscle group is large and instantaneous when the elbow is flexedduring the Normal condition. Furthermore, after elbow flexion duringthe Dead condition, the muscle group responsible for elbow joint extension performs concentric contraction when extending the elbow to take the next ladder step.

To be consistent with the sEMG results, the integral value was calculated in the motion time also in the muscle activity in Anybody, and the magnitude relation was compared.
Consequently, Normal condition (0.49 [-])> Dead condition $(0.24[-])$ in the left biceps brachialis muscle of subject A, Normal condition $(1.51[-])>$ Dead condition $(0.31[-])$ in the right biceps brachii muscle of subject $\mathrm{B}$, Normal condition (0.04 [-]) <Dead condition (0.09 [-]) in the right triceps brachii muscle of subject B was found, and the same trend as the test result was seen.

\section{DISCUSSION}

From the results in Fig. 4, it can be seen that the Dead condition results in shorter muscle activity time than Normal condition does. From this, it can be said that during the Normal condition, the climbing motion is performed sustained muscle activity.

\section{A. Nonamputee (Subject A)}

The Dead condition required significantly lower muscle activity of the biceps brachialis (elbow joint flexion muscle). From this, it was shown that in the case of subject A who is a nonamputee, Dead could reduce the loadplaced on the upper limbs. However, when comparing the results of Pmt in Fig. 5 and 6 , the muscle activity of the elbow joint flexion muscle group for approximately the same periodis performed in both conditions, and in Dead condition the load was not considered to be small. As we look closely at this, the muscle activity of the elbow joint flexion muscle group is performed at the same time in the Normal condition of Fig.5, whereas in the Dead condition of Fig.6, the muscle activity of elbow joint flexion muscle group is performed while shifting little by little at each time. This is because it is possible to express more detailed muscle activity by using musculoskeletal simulation. In sENG, because each muscle involved in elbow joint flexion is close, it is impossible to explicitlymeasure muscle activity caused by only one muscle when one muscleonly is to be measured. Therefore, it seems that it was measured as an elbow joint flexion muscle group in the experiment. In the musculoskeletal model, since it is possible to measure the muscle activity performed by only one muscle, it is thought that the biceps brachialis muscle, brachialis muscle, brachioradialis muscle of elbow joint flexion muscle group performed muscle activity at different time intervals. It can be inferred from the duration of muscle activityand \%MVC of sEMG and the duration of muscle activity of the muscle group responsible for elbow joint flexion are almost equal. Based on this, when considering muscle activity required forelbow joint flexure, contractions are performed at the same time and areshorter than that observed in the Normal condition. When this muscle activity is being performed, the action of pulling the arm by Dead is being performedsimultaneously, and nearly instantaneously. In comparison, since muscle activity was continuously performed in the Normal condition, it is considered that the muscle activity of the biceps brachii muscle was significantly greaterin Table 1 .

\section{B. Lower-limb amputee (Subject B)}

The sEMG observed in the Dead condition was significantly smaller in the biceps brachialis muscle (elbow 
joint flexion muscle); however,in terms of the brachial triceps muscle (elbow joint extension muscle) was significantlygreater. From this, it was shown that in the case of subject B who is a lower-limb amputee, Dead can reduce theload placed on the flexion muscles of the upper limbs;however, this results in an increase ofload on the extension muscles. Comparing the results of Pmt (Fig. 7 and 8 ), it was found that in Normal condition the muscle activity for the concentric contraction of the elbow joint flexion muscle group was clearlylarger than the Dead conditionduring elbow flexion, and resulted in less activity of the biceps brachii muscle during theDead condition (Table 2). ThePmtresults suggetthat the muscle activity for the eccentric contraction of the elbow joint extension muscle group is large and instantaneous at the timing when the elbow is flex in Normal condition. In addition, after flexing the elbow in the Dead condition, the muscle group responsible for elbow joint extension performed concentric contraction when extending the elbow to take the next ladder step. In Table 2, it is thoughtthat this characteristic is the reason why subject $\mathrm{B}$ significantly increased the triceps brachii muscle at Dead condition. In other words, the lower-limb amputee not only climbs using his/her arms (flexes the elbow) during the Dead technique but also pushes the arms (extend the elbow) after pulling;therefore, it can be said that muscle activity of the elbow joint extension muscle group increased. However, as described in a previous report, an eccentric contraction is known to have a higher muscle load than concentric contraction;therefore, the action of elbow extension, which is a concentric contraction is not assumed to affect the fatigue of the body[16], significantly.

Bearing these results in mind, it is likely that using Dead, reduced muscle load of upper limb during climbing, making Dead an effectivemaneuver for lower-limb amputees and nonamputees.

One potential limitation of this investigation is the lack of consideration for muscle activity of the lower limbs during climbing. There is a possibility that muscles of the lower limbs supplement the relieved load of the upper limb muscle at the time of Dead. And for lower-limb amputees, we think that the load of the lower limb muscle will be greater than that of nonamputees. Therefore, it is necessary to analyze the climbing movement focusing on the relationship between the upper limb muscle and the lower limb muscle in the future. And it is not enough for asserting the results as the number of subjects is one by one. Therefore, future investigations should repeat these measurements in a greater number of participants to increase the statistical power and applicability of the findings.

\section{CONCLUSION}

In this research, we aimed to reduce the load placed on the upper limb muscle during climbing, and by comparing Normal and Dead, we confirm the effects of Dead on nonamputee and lower-limb amputee.

In the present investigation, we studied nonamputees climbing a ladder that imitated the wall-climbing process and compared the measurements made during Normal and Dead climbing conditions. The results suggest that using Dead reduces muscle load placed on the upper limb during climbing;therefore, Dead iseffective for nonamputees. Moreover, since similar results were also obtained in lower-limb amputees, Dead iseffective for lower-limb amputees.

\section{ACKNOWLEDGMENTS}

The authors would like to thank the participants who cooperated in the experiment.

\section{REFERENCES}

[1] S. Mizumura, et al., "Supotu Climbing Kyogi ni okeru KokyoShisetu no Jyuyosei (Importance of public facilities in sport climbing competition)," Meijidaigakukyoyoronsyu Tukan509, pp.91-116, 2015. (in Japanese)

[2] INTERNATIONAL FEDERATION OF SPORT CLIMBING (iFSC), Key Figures, 2018 ,

https://www.ifsc-climbing.org/index.php/media-centre/key-figures-2, (accessed 2018-12-10).

[3] Japan ParaClimbing Association (JPCA), "ParaClimbing Toha (What is ParaClimbing)," 2018, https://www.jpca-climbing.org/blank, (accessed 2018-12-10). (in Japanese)

[4] Japan Mountaineering and Sport Climbing Association, "Climbing Kounin Ruto Setta Kitei (Climbing official recognition route setter regulations)," 2015. (in Japanese)

[5] G. Okuya, "Setudansya no Supotu Shido-Bouldering He no approach(Sports instruction of amputee - Approach to Bouldering -), " Dai24ka Nihon Gishi Sogushi Kyokai Gakujyutu Taikai, 2017. (in Japanese)

[6] T. Kiduka, et al., "Kinkatudoryo kara Mitumorareru Climbing Kei Yugu no Yukosei (Efficiency of climbing system play equipment estimated from muscle activity)," Hatuiku Hattatu Kenkyu Dai34Go, pp.1-9, 2007. (in Japanese)

[7] J. Balas, B. Strejcová, T. Malý, L. Malá, A. J.Martin, "Changes in upper body strength and body composition after 8 weeks indoor climbing in youth," Isokinetics Exercise Sci., vol. 17, Jan.2009, pp. 173-179.

[8] K. Iwasaki, G. Okuya, N. Chijiwa, H. Arizono, C.Wada, "Relationship between body acceleration and muscle load of upper limbs in lower-limb amputees during ladder climbing," 2018 International Conference on Information and Communication Technology Robotics (ICT-ROBOT), 2018.

[9] H. Higashi (2017), "Suposu Climbing Kyohon (Sport climbing textbook)," Yama to Keikokusya. (in Japanese)

[10] J. Long (1994) Gym climb!, Falcon, Guilford, CT

[11] J. Long (1997) Sport climbing, 3rd edn. Falcon, Guilford, CT

[12] K. F. Franz, G.Niegl, "Biomechanics of the two-handed dyno technique for sport climbing," Sports Eng., vol.13, Oct. 2010, pp.19-30.

[13] K. Hase, "Motion analysis by SIMM, ARMO, Any Body (SIMM, ARMO, AnyBody ni yoru Dosa Kaiseki),"Society of Biomechanisms Japan, vol.33, no.3, pp.205-211, 2009. (in Japanese)

[14] M.Damsgaard, J.Rasmussen, S.T.Christensen, E.Surma, M.De Zee, "Analysis of musculoskeletal systems in the AnyBody Modeling System," Simul. Model Pract. Theory, vol.14, Nov. 2006, pp.1100-1111.

[15] R. Nakamura, H. Saito, “Kiso Undogaku (Fundamental Kinesiology)," Ishiyaku Publishers, INC, 2000.(in Japanese)

[16] D. J.Newham, G. McPhail,K. R. Mills, R. H. Edwards, "Ultrastructural changes after concentric and eccentric contractions of human muscle," J Neurol Sci, vol.61, Sep. 1983, pp.109-122.

Kisuke Iwasaki received his A.Eng. degree in Information, Communication and Electronic Engineering from National Institute of Technology, Kumamoto College, Kumamoto, Japan, in 2015 and his B.Eng. degree in Wellbeing Science and Assistive Technology from Oita University, Oita, Japan, in 2017. He is currently a M.S. student of the Graduate School of Life Science and System Engineering, Kyushu Institute of Technology, Japan. Gooner. His research interests include ergonomics, sports engineering and assistive technology for people with disabilities. 
Chikamune Wada received the B.Eng. degree in mechanical engineering from the Osaka University, Japan, in 1990 and the Ph.D. degree in biomedical engineering from Hokkaido University, Japan, in 1996. From 1996 to 2001, he was an Assistant Professor with the Sensory Information Laboratory, in Hokkaido University. In 2001, he became an Associate Professor with Human-function Substitution System Laboratory, Kyushu Institute of Technology. Since 2016, he has been a Professor with Human-function Substitution System Laboratory. His research interestsinclude assistive technology, especially measuring human motion and informing the disabled people of the necessary information to improve their QOLs. He is a senior member of the Institute of Electronics, Information and Communication Engineers (IEICE). 